\title{
Clinical Features and Management of Endometriosis among Patients with MRKH and Functional Uterine Remnants
}

\author{
Weijie Tian NaChen Ze Liang Shuang Song Yuan Wang Yang Ye \\ Jiali Duan Lan Zhu
}

Department of Obstetrics and Gynaecology, State Key Laboratory of Complex Severe and Rare Diseases, Peking Union Medical College Hospital, Peking Union Medical College and Chinese Academy of Medical Sciences, National Clinical Research Center for Obstetrical and Gynecological Diseases, Beijing, China

\section{Keywords}

Mayer-Rokitansky-Küster-Hauser syndrome · Uterine remnants · Endometriosis

\begin{abstract}
Objective: This study aimed to determine the clinical features of Mayer-Rokitansky-Küster-Hauser (MRKH) syndrome patients with functional uterine remnants and endometriosis in a large cohort of Chinese patients. Design: This was a retrospective study. Participants: This study had a cohort of $511 \mathrm{MRKH}$ patients. A total of $34 \mathrm{MRKH}$ patients with functional remnant were finally included. Setting: This study included a tertiary referring hospital in China. Methods: Patients with MRKH diagnosed and treated at Peking Union Medical College Hospital from January 2009 to January 2020 were recruited. A cohort of $511 \mathrm{MRKH}$ patients were retrospectively screened, and a total of $34 \mathrm{MRKH}$ patients with functional remnant were finally included. Relevant clinical data were reviewed retrospectively from medical charts. $\boldsymbol{R} \boldsymbol{e}-$ sults: Of 34 patients with MRKH and functional uterine remnants, 23 (68\%) had endometriosis. These patients had a
\end{abstract}

karger@karger.com www.karger.com/goi

Karger $\frac{1}{\%}$
(C) 2021 The Author(s)

Published by S. Karger AG, Basel

This is an Open Access article licensed under the Creative Common Attribution-NonCommercial-4.0 International License (CC BY-NC) (http://www.karger.com/Services/OpenAccessLicense), applicable to the online version of the article only. Usage and distribution for commercial purposes requires written permission. greater mean age at MRKH diagnosis than patients without endometriosis ( $15.9 \pm 3.3$ years vs. $13.2 \pm 3.5$ years; $p=0.03$ ). Similarly, these patients experienced a longer time between age at onset of symptoms and age at operation than patients without endometriosis $(45.5 \pm 39.6$ years vs. $19.8 \pm 13.2$ years; $p=0.04)$. In addition, the CA125 level was significantly higher in patients with endometriosis than in those without it $(64.9 \pm 85.9 \mathrm{U} / \mathrm{mL}$ vs. $25.5 \pm 19.1 \mathrm{U} / \mathrm{mL} ; p=0.03)$. Limitations: The number of patients with MRKH analyzed in this study was low as we restricted inclusion to patients with at least 1 functional uterine remnant or endometriosis. Conclusions: It is reasonable to monitor the uterine remnant of patients with MRKH closely, regardless of age, to achieve early intervention. The level of CA125 might be helpful to differentiate active uterine remnants with endometriosis and schedule individualized treatments.

2021 The Author(s)

Published by S. Karger AG, Basel

Weijie Tian and $\mathrm{Na}$ Chen should be considered similar in the author order of the study.
Correspondence to:

Lan Zhu, zhu_julie@vip.sina.com 


\section{Introduction}

Mayer-Rokitansky-Küster-Hauser (MRKH) syndrome (also known as Müllerian aplasia) results from disrupted development of the Müllerian ducts. It is characterized by agenesis or hypoplasia of the uterus and vagina with normal secondary sex characteristics in females. Despite the absence of a normal uterus, approximately $40 \%$ of affected patients with uterine remnants have some endometrium $[1,2]$. About $50 \%$ of these can experience cyclic pelvic pain-like menstrual symptoms but without bleeding [1]. Continuous bleeding in the uterine remnant might form hematometra and lead to reflux of blood to the peritoneal cavity through the ipsilateral Fallopian tube.

Endometriosis is defined as the presence of endometrial tissue outside the internal epithelial lining of the uterine cavity. It is the leading pathologic cause of dysmenorrhea and chronic pelvic pain among adolescents [3]. Several theories have been proposed to account for the pathogenesis of endometriosis, but only retrograde menstruation followed by the endometrial tissue implanting on surfaces in the pelvic cavity is widely accepted as the leading cause $[4,5]$.

Of note, obstructive Müllerian duct anomalies showed a strong association with endometriosis [6]. The deranged peristalsis and tubal reflux menstruation of obstructive anomalies constitute risk factors for endometriosis $[7,8]$. Following the theory of retrograde menstrual flow as an etiology of endometriosis, several studies have demonstrated an increased risk of endometriosis in patients with obstructive genital anomalies $[9,10]$. However, in those studies, patients with endometriosis were not stratified based on the types of deformities.

MRKH syndrome with functional uterine remnant is a rare subtype of such obstructive anomalies. During the earliest stages of embryogenesis, failure of Müllerian duct development results in a functioning endometrium that is not connected to a normal outflow tract. Thus, functional remnants causing cyclic pelvic pain might result in endometriosis and severely affect adolescents' quality of life.

Several case studies have presented endometriosis in patients with MRKH [11-13]. However, these case reports with low numbers of patients were not sufficient to capture the features of endometriosis in patients with MRKH. Thus, the exact nature of the relationship between MRKH with functional remnant and endometriosis has yet to be elucidated, and a systematic evaluation is necessary. Here, we systematically analyzed the clinical

Functional Uterine Remnants of MRKH Patients features and management of functional uterine remnants and associated endometriosis in a large cohort of patients with MRKH.

\section{Materials and Methods}

Study Design and Population

Patients with MRKH diagnosed and treated at the Department of Obstetrics and Gynecology, Peking Union Medical College Hospital (PUMCH), Chinese Academy of Medical Sciences, from January 2009 to January 2020 were recruited. The inclusion criteria were as follows: (1) normal karyotype of 46, XX; (2) uterovaginal agenesis or uterine remnant verified by pelvic ultrasonography or magnetic resonance imaging; (3) normal female secondary sexual characteristics; (4) undergone surgery for excising uterine remnants because of possible functional remnants or adnexal mass or vaginoplasty; and (5) pathohistological confirmed endometriosis or functional endometrium. The exclusion criteria were as follows: (1) abnormal karyotypes and (2) cervical atresia, vaginal atresia, or transverse vaginal septum with hematometra and hematocolpos.

Relevant clinical data were reviewed retrospectively from charts of recruited patients. Endometriosis diagnosed at the time of surgery was staged in accordance with the revised American Society for Reproductive Medicine (rASRM) guidelines [14]. Follow-up ended with the last inpatient or outpatient contact after surgery.

\section{Statistical Analyses}

Statistical analysis between groups was carried out using $\chi^{2}$, Fisher's exact, and Student's $t$ tests where appropriate. Statistical analyses were performed using GraphPad Prism software version 8.0 (GraphPad Software, San Diego, CA, USA). The significance level for all analyses was set at $p<0.05$.

Statement of Ethics

The study was approved by the Ethics Committee of Peking Union Medical College Hospital (ZS-1858). All subjects have given their written informed consent for this review of clinical data.

\section{Results}

In total, 511 patients were diagnosed with $\mathrm{MRKH}$ at PUMCH from January 2009 to January 2020. Of these, 375 were treated with dilation as outpatients and 136 underwent vaginoplasty or excision of uterine remnants because of possible functional remnants or an adnexal mass. Of the 136 charts reviewed, 57 patients underwent peritoneal vaginoplasty without excision of uterine remnants and 45 did not have functional endometrium or endometriosis confirmed by pathology; finally, 34 patients with MRKH conformed to our inclusion criteria (Fig. 1).

All 34 patients underwent excision of uterine remnants and ipsilateral salpingectomy because of possible 
Fig. 1. The flowchart of this study. MRKH, Mayer-Rokitansky-Küster-Hauser syndrome.

Table 1. Clinical characteristics of patients with MRKH and functional uterine remnants

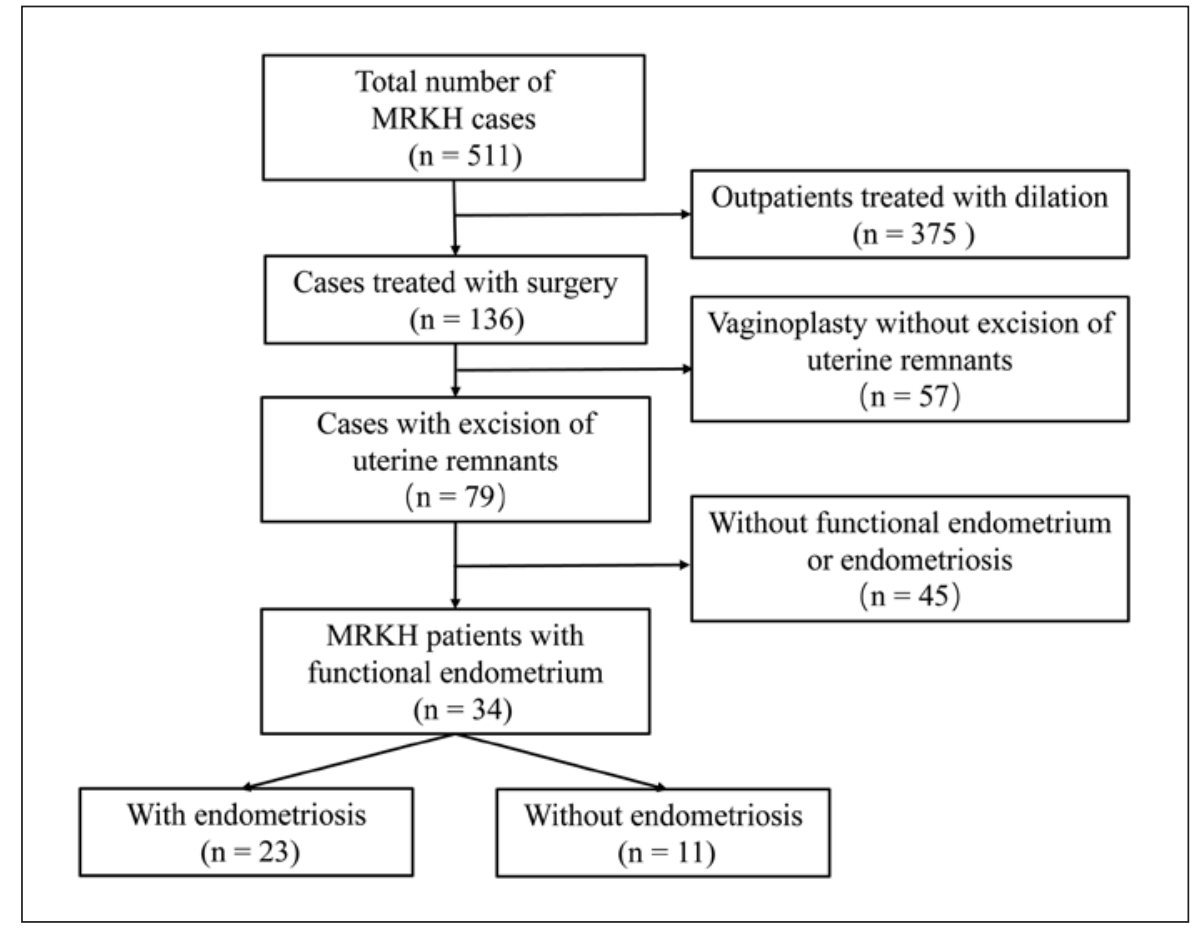

\begin{tabular}{ll}
$n$ & 34 \\
Age at MRKH diagnosis, yr & $15.0 \pm 3.5(5-26)$ \\
Duration between age at onset of symptoms and age & \\
$\quad$ at operation, mo & $37.2 \pm 35.3(0.5-144)$ \\
VAS score & $6.1 \pm 2.1(0-9)$ \\
CA125, U/mL & $49.4 \pm 69.9(8.8-378.2)$ \\
Age at operation & $18.1 \pm 4.8(12-29)$ \\
Cyclic pain & $30(88)$ \\
Site of remnant & \\
$\quad$ Bilateral & $34(100)$ \\
Functional remnant & \\
$\quad$ Left & $14(41)$ \\
$\quad$ Right & $9(27)$ \\
$\quad$ Bilateral & $11(32)$ \\
Kidney agenesis & 8 \\
Skeletal malformations & 7 \\
Other malformations & 6 \\
Hysterectomy + salpingectomy & 34 \\
Vaginoplasty & 11 \\
Follow-up, mo & $46.3 \pm 22.8(15.2-92.5)$ \\
\hline
\end{tabular}

Values are presented as mean \pm SD (range) or $n(\%)$. yr, year; mo, month; MRKH, MayerRokitansky-Küster-Hauser syndrome. functional remnants or an adnexal mass, and $11(32 \%)$ of them received peritoneal vaginoplasty simultaneously. No patients were diagnosed with endometriosis without a functional remnant. The 34 patients had a mean age at MRKH diagnosis of $15.0 \pm 3.5$ years (range 5.0-26.0) and a mean age at operation of $18.1 \pm 4.8$ years (range $12-29$ ). Pelvic pain was common in these patients, with $30 / 34$ (88\%) describing their pain as cyclic and $4 / 34(12 \%)$ as acyclic. The mean visual analog scale (VAS) pain score was $6 \pm 2.1$ (range $0-9$ ), the mean serum carcinoma anti- 
(A)

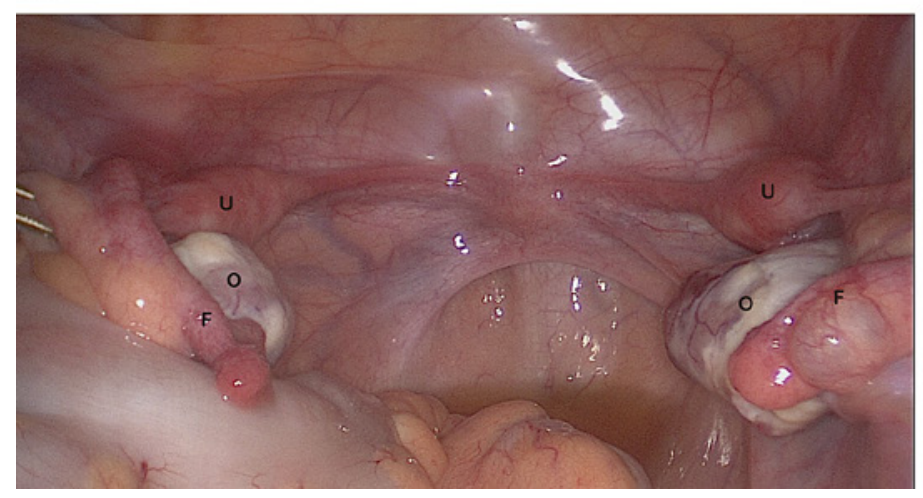

(B)

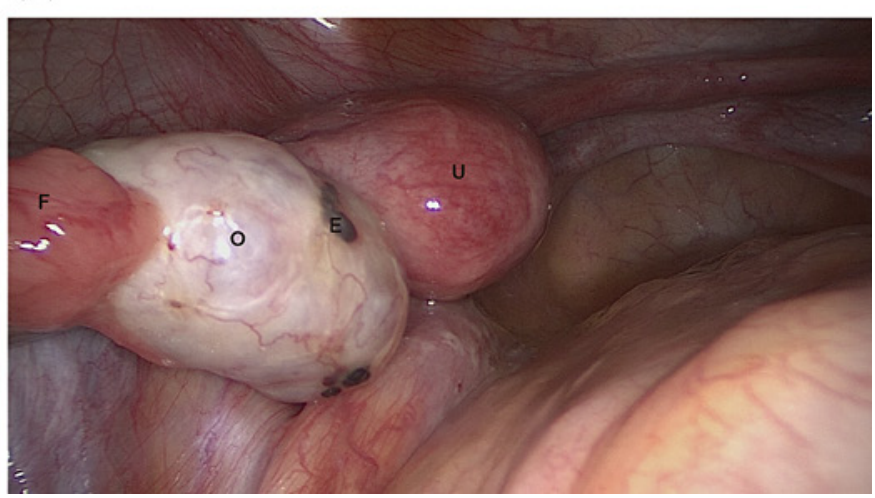

Fig. 2. Internal genital organs of patients with MRKH. A patient with left functional remnant but without endometriosis (a); a patient with left functional remnant and endometriosis (b). MRKH, Mayer-Rokitansky-KüsterHauser syndrome; U, uterine remnant; O, ovary; F, fallopian duct; E, endometriosis.

gen (CA) 125 level was $49.4 \pm 69.9 \mathrm{U} / \mathrm{mL}$ (range: $8.8-$ $378.2 \mathrm{U} / \mathrm{mL}$ ), and the mean time between the patient age at onset of symptoms and that at operation was $37.2 \pm$ 35.3 months (range 0.5-144; Table 1). All 34 patients had bilateral uterine remnants; 11 (32\%) patients had 2 functional remnants, $14(41 \%)$ patients had a functional left uterine remnant, and $9(27 \%)$ patients presented with a functional right uterine remnant. Half (17/34) of the patients had various concomitant malformations, including 8 patients with unilateral renal agenesis $(2$ complicated with skeletal deformities and 2 complicated with anal atresia), 7 with skeletal malformations ( 4 with idiopathic scoliosis and 3 with limb malformations), and 6 with other malformations ( 3 cases of anal atresia and 3 of cardiac malformations). The mean follow-up time was $46.3 \pm$ 22.8 months (range 15.2-92.5).

Of the $34 \mathrm{MRKH}$ patients with functional endometrium, 23 (68\%) were diagnosed with endometriosis and 11 (32\%) without (Fig. 2). Patients with pelvic endometriosis had a greater mean age at MRKH diagnosis than those without it ( $15.9 \pm 3.3$ years vs. $13.2 \pm 3.5$ years; $p=0.03$ ) Similarly, patients with endometriosis experienced a longer time between their age at the onset of symptoms and their age at operation than patients without it $(45.5 \pm 39.6$ years vs. $19.8 \pm 13.2$ years; $p=0.04)$. In addition, the CA125 level was significantly higher in patients with endometriosis than in those without it $(64.9 \pm 85.9 \mathrm{U} / \mathrm{mL}$ vs. $25.5 \pm 19.1 \mathrm{U} / \mathrm{mL} ; p=0.03)$. The VAS score and the age at operation had no statistical difference between the 2 groups. However, there was a tendency that patients with endometriosis reported a higher VAS pain score $(6.2 \pm 1.8$ vs. $5.9 \pm 2.6)$ and greater age at operation $(19.0 \pm 5.2$ vs.
$16.2 \pm 3.3$ years). There was also no significant difference in the distribution of functional uterine remnants between the 2 groups (Table 2).

Regarding the 23 patients with MRKH and endometriosis, 15 had endometrioma with a mean size of $4.53 \mathrm{~cm}$ (range 1-9), 4 had adenomyosis, and 9 presented with superficial peritoneal lesions (of these patients, 5 had endometrioma simultaneously). No case of deeply infiltrating endometriosis was found. All patients with endometriosis had ovarian cysts or adenomyosis ipsilateral to the functional remnant. The mean rASRM score of all patients was $27.4 \pm 21.2$ (range 1-76), and 14 (74\%) patients demonstrated rASRM stage III-IV endometriosis. All patients were discharged uneventfully, and none complained of recurrent pelvic pain or recurrence of endometriosis at the last follow-up (range 15.2-92.5 months).

\section{Discussion}

Here, to our knowledge, we report the largest cohort of MRKH patients with functional uterine remnants and related endometriosis. The principal finding was that $68 \%$ of the $34 \mathrm{MRKH}$ patients with functional remnants also suffered endometriosis. In addition, these patients had a greater mean age at MRKH diagnosis, experienced a longer time between age at onset of symptoms and age at operation, and had a greater CA125 level than patients without endometriosis.

All the patients in this study had bilateral uterine remnants, but most (68\%) of them had only 1 functional remnant. Müllerian duct development during embryogenesis 
Table 2. Clinical characteristics of patients with MRKH with functional uterine remnants and associated endometriosis

\begin{tabular}{lll}
\hline & $\begin{array}{l}\text { With } \\
\text { endometriosis }\end{array}$ & $\begin{array}{l}\text { Without } \\
\text { endometriosis }\end{array}$ \\
\hline$n$ & $23(68)$ & $11(32)$ \\
Age at MRKH diagnosis, yr & $15.9 \pm 3.3^{*}$ & $13.2 \pm 3.5$ \\
Duration between age at onset of symptoms and age at operation, mo & $45.5 \pm 39.6^{*}$ & $19.8 \pm 13.2$ \\
VAS score & $6.2 \pm 1.8$ & $5.9 \pm 2.6$ \\
CA125, U/mL & $64.9 \pm 85.9^{*}$ & $25.5 \pm 19.1$ \\
Age at operation, yr & $19.0 \pm 5.2$ & $16.2 \pm 3.3$ \\
Cyclic pain & $20(87)$ & $10(91)$ \\
Functional remnant & & $6(55)$ \\
$\quad$ Left & $8(35)$ & $2(18)$ \\
$\quad$ Right & $7(30)$ & $3(27)$ \\
$\quad$ Bilateral & $8(35)$ & \\
Site of endometrioma & $10(44)$ & \\
$\quad$ Left & $8(35)$ & \\
$\quad$ Right & $2(9)$ & \\
$\quad$ Bilateral & $27.4 \pm 21.2(1-76)$ & \\
rASRM score (range) & & \\
rASRM stage for endometriosis & $5(26)$ & $14(74)$ \\
$\quad$ I-II & $15(65)$ & \\
$\quad$ III-IV & $4(17)$ & \\
$\quad$ Endometrioma & $9(39)$ & \\
$\quad \begin{array}{l}\text { Adenomyosis } \\
\text { Superficial peritoneal lesions }\end{array}$ & $4.53(1-9)$ & \\
\hline
\end{tabular}

Values are presented as mean \pm SD (range) or $n$ (\%). yr, year; mo, month; MRKH, Mayer-Rokitansky-Küster-Hauser syndrome; rASRM, revised American Society for Reproductive Medicine. ${ }^{*} p<0.05$ versus without endometriosis.

involves the elongation of paired ducts followed by fusion, canalization, and septal resorption [15]. The varying states of the uterus in patients with MRKH result from aberrations in this developmental process. However, the causes of MRKH syndrome remain poorly understood. Studies showed that about $30 \%$ of patients with MRKH demonstrated endometrium confirmed by histological analysis $[1,16]$. In our cohort, the proportion of MRKH cases with a functional uterine remnant $(25 \%)$ was similar to previous studies.

In our cohort, $68 \%$ of patients with MRKH with functional remnants were complicated with endometriosis. This proportion is consistent with the reported prevalence of 56\% by Marsh et al. [1]. All MRKH patients with endometriosis had a functional uterine remnant, and all had ovarian cysts or adenomyosis ipsilateral to the functional remnant. This is consistent with Sampson's retrograde menstruation theory as one of the etiologies of endometriosis [17]. Although complicated with a functional remnant, the remaining $32 \%$ of MRKH patients without endometriosis suggest that other factors might have influenced the implantation of endometrial remnants in the peritoneum. These potential additional factors include patient-specific immune characteristics, quantity of retrograde flow, or genetic predisposition $[9,18,19]$.

Here, MRKH patients with functional uterine remnants and endometriosis experienced a longer period between age at onset of symptoms and age at operation. MRKH patients usually sought medical care complaining of primary amenorrhea or abdominal pain. Although cyclic pain helps diagnose functional uterine remnants, our cohort had 3 patients with functional endometrium but without cyclic pain. Particular attention should be given to patients with $\mathrm{MRKH}$, as amenorrhea lessens the characterization of pain during menses. Awareness of the increased risk of progressive endometriosis among these patients might help improve early diagnosis, prompt early intervention, and prevent morbidity secondary to hematometra and endometriosis, such as pain and distorted anatomy. 
The treatment timing and modalities of MRKH can vary depending on whether an activated remnant or potential endometriosis exists. For patients with evidence of functional remnants, removing them is strongly recommended, both for treating pelvic pain and reducing the likelihood of endometriosis [20]. Several case reports have reported management of endometriosis in patients with MRKH. All of them underwent removal of the uterine remnants, and most reported complete remission of pain after surgery and no recurrence of endometriosis [1, 11-13, 19, 21-27]. Similarly, all our patients reported uneventful recovery after the operation, and none complained of recurrent pelvic pain or recurrence of endometriosis in the follow-up.

However, there is no common consensus on dealing with the asymptomatic remnants [28]. Thus, Enatsu et al. [22] reported a patient with MRKH diagnosed with active remnants 8 years after vaginoplasty. Deligeoroglou et al. [23] presented a patient with MRKH who underwent the creation of a neovagina at the age of 21 . However, the patient was admitted twice to the hospital because of recurrent pelvic pain and was finally diagnosed with salpingitis and functioning endometrium. The oldest patient with MRKH presenting with functional uterine remnants was reported by Parkar et al. [11]. This asymptomatic patient demonstrated cyclic pelvic pain at the age of 40 and was diagnosed with functioning uterine remnants. Rall et al. [16] showed that the endometrium of uterine remnants in patients with MRKH had significantly low proliferative ability but normal estrogen or progesterone receptor expression levels. Because the hormonal levels are normal, the inadequate proliferative potential of the endometrium might explain the postponed bleeding of uterine remnants. In our cohort, MRKH patients with functional remnants and endometriosis had a greater age at their initial diagnosis of $\mathrm{MRKH}$. Therefore, it is reasonable to monitor the asymptomatic remnants closely regardless of age. We also found that MRKH patients with functional uterine remnants and endometriosis had higher CA125 levels. This biomarker might be helpful to differentiate such active remnants with endometriosis and schedule individualized treatment.

This study had several limitations. First, it was a retrospective one; a prospective design is not feasible when studying such rare reproductive organ anomalies. Second, all patients with MRKH were treated in a tertiary referring center for this disorder, which might have caused some selection bias. Finally, although the number of patients with MRKH analyzed in this study was low as

Functional Uterine Remnants of MRKH

Patients we restricted inclusion to patients with at least 1 functional uterine remnant or endometriosis, as far as we know, it is the largest cohort ever reported.

\section{Conclusions}

Among the $34 \mathrm{MRKH}$ patients with functional endometrium, $23(68 \%)$ also had endometriosis. These patients had a greater mean age at MRKH diagnosis, experienced a longer time between age at onset of symptoms and age at operation, and had a higher CA125 level than patients without endometriosis. Therefore, it is reasonable to closely monitor the uterine remnant of patients with MRKH regardless of their age for early intervention. The level of CA125 might be helpful to differentiate active remnants with endometriosis and plan individualized treatments.

\section{Acknowledgments}

We thank all the patients, families, and physicians involved in the study. We also thank James M., Cummins D. from Liwen Bianji (Edanz) (www.liwenbianji.cn/ac) for editing the English text of a draft of this manuscript.

\section{Statement of Ethics}

The study was approved by the Ethics Committee of Peking Union Medical College Hospital (ZS-1858). All subjects have given their written informed consent for this review of clinical data.

\section{Conflict of Interest Statement}

The authors declare that no conflicts of interest could be perceived as prejudicing the impartiality of this research.

\section{Funding Sources}

This research was funded in part by the CAMS Initiative for Innovative Medicine CAMS-2017-I2M-1-002 to Z.L., National Science Foundation for Youth, 81801401 to C. N., and National Key Science Foundation, 81830043 to Z.L.

\section{Author Contributions}

W.J.T. performed the data analyses and drafted and revised the manuscript. N.C. reviewed and corrected the manuscript. L.Z. contributed to the manuscript revision. Z.L., S.S., Y.W., Y.Y., and 
J.L.D. helped with data acquisition and performed the analysis with constructive discussions. W.J.T. and N.C. contributed to manuscript preparation. L.Z. supervised and approved the final version of the manuscript.

\section{Data Availability Statement}

Data are available upon reasonable request. The datasets analyzed during the current study are available from the corresponding author on reasonable request.

\section{References}

1 Marsh CA, Will MA, Smorgick N, Quint EH, Hussain H, Smith YR. Uterine remnants and pelvic pain in females with Mayer-Rokitansky-Küster-Hauser syndrome. J Pediatr Adolesc Gynecol. 2013 Jun;26(3):199-202.

2 Preibsch H, Rall K, Wietek BM, Brucker SY, Staebler A, Claussen CD, et al. Clinical value of magnetic resonance imaging in patients with Mayer-Rokitansky-Küster-Hauser (MRKH) syndrome: diagnosis of associated malformations, uterine rudiments and intrauterine endometrium. Eur Radiol. 2014 Jul; 24(7):1621-7.

3 ACOG committee opinion No. 760: dysmenorrhea and endometriosis in the adolescent. Obstet Gynecol. 2018 Dec;132(6):e249-e58.

4 Sampson JA. Peritoneal endometriosis due to the menstrual dissemination of endometrial tissue into the peritoneal cavity. Am J Obstet Gynecol. 1927;14(4):422-69.

5 Burney RO, Giudice LC. Pathogenesis and pathophysiology of endometriosis. Fertil Steril. 2012;98(3):511-9.

6 Kaya C, Cengiz H, Ekin M, Yasar L. Transverse vaginal septum: a benign reason for elevated serum CA 19-9 and CA 125 levels. Arch Gynecol Obstet. 2012 Sep;286(3):821-3.

7 Leyendecker G, Kunz G, Herbertz M, Beil D, Huppert P, Mall G, et al. Uterine peristaltic activity and the development of endometriosis. Ann N Y Acad Sci. 2004;1034:338-55.

8 Freytag D, Mettler L, Maass N, Günther V, Alkatout I. Uterine anomalies and endometriosis. Minerva Med. 2020 Feb;111(1):33-49.

9 Olive DL, Henderson DY. Endometriosis and mullerian anomalies. Obstet Gynecol. 1987 Mar;69(3 Pt 1):412-5.

10 Uğur M, Turan C, Mungan T, Kuşçu E, Senöz S, Ağiş HT, et al. Endometriosis in association with müllerian anomalies. Gynecol Obstet. 1995;40(4):261-4.
11 Parkar RB, Kamau WJ. Images in endoscopy. Laparoscopic excision of bilateral functioning noncommunicating and rudimentary uterine horns in a patient with Mayer-RokitanskyKüster-Hauser syndrome and pelvic endometriosis. J Minimal Invas Gynecol. 2009 Sep; 16(5):522-4.

12 Mok-Lin EY, Wolfberg A, Hollinquist $\mathrm{H}$, Laufer MR. Endometriosis in a patient with Mayer-Rokitansky-Küster-Hauser syndrome and complete uterine agenesis: evidence to support the theory of coelomic metaplasia. J Pediatr Adolescent Gynecol. 2010;23(1):e35e37.

13 Elliott JE, Abduljabar H, Morris M. Presurgical management of dysmenorrhea and endometriosis in a patient with Mayer-Rokitansky-Kuster-Hauser syndrome. Fertil Steril. 2011 Aug;96(2):e86-9.

14 Revised American society for reproductive medicine classification of endometriosis: 1996. Fertil Steril. 1997 May;67(5):817-21.

15 Atsuta Y, Takahashi Y. Early formation of the Müllerian duct is regulated by sequential actions of BMP/Pax2 and FGF/Lim1 signaling. Development. 2016 Oct 1;143(19):3549-59.

16 Rall K, Barresi G, Wallwiener D, Brucker SY, Staebler A. Uterine rudiments in patients with Mayer-Rokitansky-Küster-Hauser syndrome consist of typical uterine tissue types with predominantly basalis-like endometrium. Fertil Steril. 2013 Apr;99(5):1392-9.

17 Sampson JA. Heterotopic or misplaced endometrial tissue. Am J Obstet Gynecol. 1925; 10(5):649-64.

18 Kaya C, Alay I, Guraslan H, Gedikbasi A, Ekin $M$, Ertaş Kaya S, et al. The role of serum caspase 3 levels in prediction of endometriosis severity. Gynecol Obstet Inves. 2018;83(6): 576-85.

19 Konrad L, Dietze R, Kudipudi PK, Horné F, Meinhold-Heerlein I. Endometriosis in MRKH cases as a proof for the coelomic metaplasia hypothesis?. Reproduction. 2019; 158(2):R41-7.
20 Acién P, Acién M, Sánchez-Ferrer M. Complex malformations of the female genital tract. New types and revision of classification. Hum Reprod. 2004;19(10):2377-84.

21 Acien P, Lloret M, Chehab H. Endometriosis in a patient with Rokitansky-Kuster-Hauser syndrome. Gynecol Obstet Invest. 1988;25(1): 70-2.

22 Enatsu A, Harada T, Yoshida S, Iwabe T, Terakawa $\mathrm{N}$. Adenomyosis in a patient with the Rokitansky-Kuster-Hauser syndrome. Fertil Steril. 2000 Apr;73(4):862-3.

23 Deligeoroglou E, Christopoulos P, Creatsas G. A unique case of descending salpingitis and functioning endometrium in a Müllerian remnant in a woman with Mayer-Rokitansky-Küster-Hauser syndrome. Fertil Steril. 2005 May;83(5):1547-8.

24 Yan L, Zhao X, Qin X. MRKH syndrome with endometriosis: case report and literature review. Eur J Obstet Gynecol Reprod Biol. 2011 Nov; 159(1):231-2.

25 Kawano Y, Hirakawa T, Nishida M, Yuge A, Yano M, Nasu K, et al. Functioning endometrium and endometrioma in a patient with Mayer-Rokitanski-Kuster-Hauser syndrome. Jpn Clin Med. 2014;5:43-5.

26 Troncon JK, Zani AC, Vieira AD, Poli-Neto OB, Nogueira AA, Rosa-E-Silva JC. Endometriosis in a patient with mayer-rokitanskyküster-hauser syndrome. Case Rep Obstet Gynecol. 2014;2014:376231.

27 Naem A, Shamandi A, Al-Kurdy B. A rare form of Mayer-Rokitansky-Küster-Hauser syndrome associated with ovarian endometrioma: a case report. J Surg Case Rep. 2020 Sep;2020(9):rjaa393.

28 ACOG committee opinion No. 728: müllerian agenesis: diagnosis, management, and treatment. Obstet Gynecol. 2018;131(1):e35e42. 